\title{
Clavien-Dindo Grade IIIb
}

National Cancer Institute

\section{Source}

National Cancer Institute. Clavien-Dindo Grade IIIb. NCI Thesaurus. Code C121451.

An intervention under general anaesthesia. 